\title{
Airline ticket reservation using NFC-based single identity
}

\author{
Mikhael Bagus Renardi', Noor Cholis Basjaruddin ${ }^{2}$, Supriyadi ${ }^{3}$, Kuspriyanto ${ }^{4}$ \\ ${ }^{1,4}$ School of Electrical Engineering and Informatics, Bandung Institute of Technology, Bandung, Indonesia \\ ${ }^{2,3}$ Department of Electrical Engineering, Bandung State Polytechnic, Bandung, Indonesia
}

\begin{tabular}{l}
\hline Article Info \\
\hline Article history: \\
Received Oct 11, 2018 \\
Revised Nov 17, 2018 \\
Accepted Nov 30, 2018 \\
\hline
\end{tabular}

Keywords:

Airline Ticketing

Near Field Communication

Single Identity

Ticket Reservation

\begin{abstract}
Airline ticket reservations can be completed via ticket counters or online. To reduce passenger data redundancy, Single Identity (SI) may be required to input passenger data. In Indonesia, SI is utilised in identity cards using Near Field Communication (NFC), called electronic Kartu Tanda Penduduk (eKTP). However, the use of e-KTP is not optimal yet because it is still used in the same way as the conventional one. Data input using SI can be done by tapping the e-KTP on a NFC-enabled machine, and the user is required to select the passengers' names. The process of data input may be simplified, resulting in a higher transaction speed. This research result demonstrated that there was a slight difference of the time required between the two methods for ticket reservations. The time efficiency using SI through ticket counters was $48 \%$, while the online reservation using SI was $45 \%$. Further research may implement the validation methods, such as using PIN or fingerprint.
\end{abstract}

Copyright (C) 2019 Institute of Advanced Engineering and Science. All rights reserved.

\section{Corresponding Author:}

Noor Cholis Basjaruddin, Department of Electrical Engineering,

Bandung State Polytechnic,

Jl. Gegerkalong Hilir Ds.Ciwaruga Bandung 40012.

Email: noorcholis@polban.ac.id

\section{INTRODUCTION}

Single Identity (SI) has been implemented particularly in Indonesia for the purpose of demographic data integration and update [1]. SI contains an individual's identity including full name, gender, occupation, address, marital status, date and place of birth, and blood type [2]. The making of SI numbers may also reveal one's data such as, region, place and date of birth, and the number of residents having the same birth data in a particular area. SI numbers are permanent, meaning that they are used by an individual from birth to death. Moreover, SI is utilised in an electronic Kartu Tanda Penduduk (e-KTP) (electronic identity card), Birth Certificate, and Family Card [3]. The utilisation of e-KTP is considered to be inefficient because it is still used in the same way as the conventional one such as, the card is photocopied and the data in the e-KTP are still checked manually.

One of the public services utilising SI is transport services, especially airlines [4]. SI is used to ensure whether a passenger identity matches the data attached in the ticket or in the booking reference. Identity input is done when a passenger purchases airline tickets, while validation is completed in the check-in process. The validation is usually done by confirming the data in the ticket with the data in the e-KTP. Data input completed manually may possibly cause data redundancy, especially passengers' names. For example, a passenger's name Mikhael Bagus Renardi written M Bagus R may refer to different individuals, but it may also refer to one individual [2], [5], [6]. Hence, SI should be utilised as a method for reference data input in order to reduce data redundancy.

Both conventional and online ticket reservations are completed by entering data manually. This process is done in each ticket reservation. Sometimes the process can be exhausting as passengers need to input the same data repeatedly. One of the drawbacks of the conventional ticket reservations is that passengers are required to visit ticket counters or places that offer ticket reservations, limited by the distance and time [7], [8]. 
Meanwhile, online ticket reservations require an internet connection to do transactions [9], [10]. One of the possible security gaps of online reservations is that there is no validation process so the data may be falsified or used by unauthorised parties.

The use of e-KTP in online ticket reservations may shorten the process of data input for passengers [2], [11], [12]. Moreover, e-KTP numbers may also be used to recognise identity numbers of other family members stated in the same Family Card, thus, the data input can be completed faster. Additionally, ticket reservations may be done by using machines, such as Automatic Teller Machine (ATM) provided in strategic areas and validation via smartphones when passengers enter their data using the NFC reader [13], [14]. Also, ticket reservation businesses should be standardised, so that the same steps can be applied in all ticketing businesses and the expected outcome can be achieved.

Nowadays, the development of smartphone technology has influenced smartphone users to integrate their work and businesses into a smartphone. Generally, some people may not favour the use of cards for each business. Thus, saving data in smartphones can be a solution because it offers an easy way of managing data storage as a substitute for the use of cards [15]. The utilisation of NFC in smartphones can be maximised by storing identity data in smartphones, so that these data can be used in transaction without the use of e-KTP.

\section{RESEARCH METHOD}

This research began with literature studies on E-KTP, conventional tickets and online tickets, and conventional and online ticket reservations. This literature study produced some information about time efficiency for online ticket reservation systems by using NFC-based SI. The results can be used to determine the effectiveness of the use of NFC for data input and for reducing data redundancy. This research on ticket reservations using SI and NFC was drawn from some studies investigating online ticket reservations and the application of SI in a wide range of areas [5-9]. Nevertheless, in some regions, the implementation of ticket reservations including online reservations does not have a uniform system. Thus, business processes and reservation methods available need to be understood comprehensively.

Some research results in this field have revealed that purchasing tickets online is more preferable due to its fast process [16]. Possible issues in an online ticketing system might be the availability of internet connection to complete transactions [17]. In addition, to ensure that data (e-KTP) users are the actual owners, scanning the e-KTP is required as a substitute for identity input. Other studies, e.g. see ref. 18, related to the application of NFC in airports have also investigated the use of NFC in baggage claims. This study has concluded that the implementation of NFC may shorten the time required in baggage claims. Other research has also examined the utilisation of NFC in Medical Record (MR) and how data security in MR can be done [19-20].

\subsection{E-KTP}

E-KTP is an identity card given to an Indonesian citizen when he/she is already mature. The e-KTP is connected to biometric data such as, fingerprint and retina scans. A citizen is only allowed to have one SI that is used as an official identification. Moreover, the e-KTP is also equipped with Near Field Communication (NFC) Tag so it can store data [21-23]. However, rarely do public and private services utilise the NFC tag in the e-KTP in their businesses [24-26]. In fact, the use of SI in various aspects of life may ease administration processes and other technical matters.

Conventional KTP is usually used by photocopying the card as required in the administrative process. In the validation process, the KTP is used as a proof that the data stated in the administrative process correspond to the data in the KTP. Unfortunately, the e-KTP is still used in the same way as the conventional one. Also, the e-KTP should not be photocopied because the chip inside the card may be broken. Rather, the data of the e-KTP holder can be retrieved by scanning the card using an NFC device [12]. Therefore, by using this method, the process of data input and data validation is done by the system, resulting in faster transactions.

\subsection{Conventional Ticket and Electronic Ticket}

Tickets are a document used to show that passengers whose name and data attached in the ticket have the right to services and facilities offered by service providers [10]. The ticket is usually made of paper which is printed out when the check-in process is completed. In some transportation service providers, the ticket can be printed out when passengers have bought the ticket. The printed ticket is usually checked and torn when passengers are about to board the airplane. Thus, the transactions may require much paper as the number of passengers may increase [4], [15].

Although nowadays tickets are in a form of electronic tickets (e-tickets), sometimes e-tickets are still used in the same way as conventional tickets (e-tickets are required to be printed and are checked manually). E-tickets also have a reference code called as a booking code. Also, passenger data, flight schedules, and flight 
destinations are stated in e-tickets. The use of e-ticket should promote paperless transactions, and the check-in process should be done online or using self-service check-in machines, thus, these may avoid a long queue and shorten the time needed for transactions [18].

\subsection{Conventional and Online Ticket Reservations}

Conventional ticket reservations mean that passengers are required to visit ticket counters in airports or other authorised parties [11]. During this process, passengers will fill in a form (or the staff will help passengers fill in the form) regarding their personal data. Passengers then inform the staff regarding the departure and arrival time and place. After that, the staff proceeds the reservation and print out the ticket or the booking reference stating transaction numbers and details of flight schedules. Meanwhile, online ticket reservations can be completed by vising related websites or by using applications for ticket reservations [15], and passengers need to fill in the form online. Passengers search and select the time and the flight destination. After passengers confirm the booking, the booking reference will be sent to their email. The flowchart of both conventional and online ticket reservation can be seen in Figure 1.

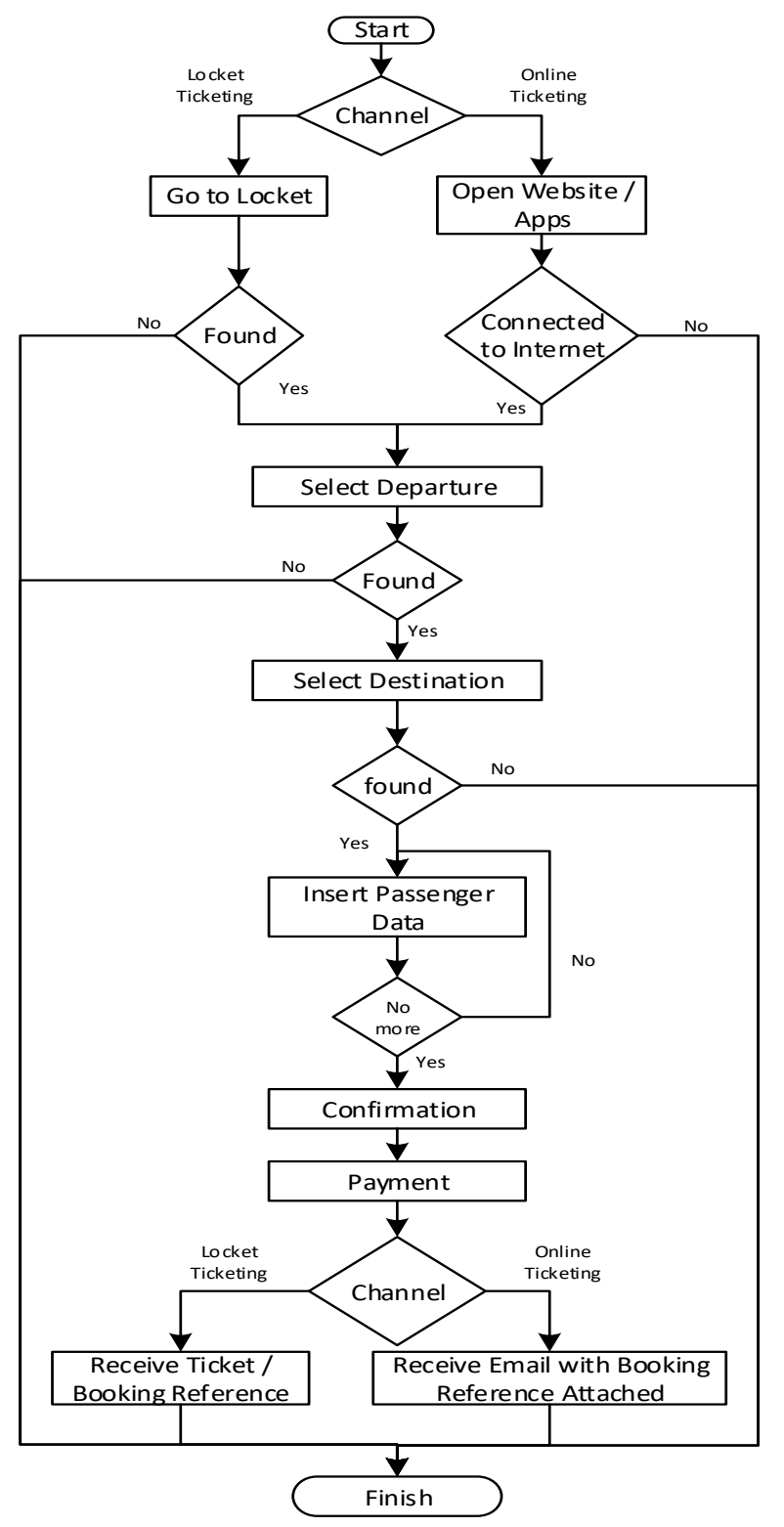

Figure 1. Flowchart of conventional and online ticket reservations 


\section{RESULTS AND ANALYSIS}

By applying SI, every piece of data of passengers and family members can be retrieved from the database. In purchasing tickets, when passengers input their data, they are required to select their family members who will also be registered. Manual data input is not used as all the data utilised are drawn from the database. Hence, the redundancy can be minimised. Additionally, passengers are not required to enter their data repeatedly as the data have been stored in the database. Another merit is that passengers do not need to concern in case they forget or do not know the personal data of their family members.

The application of SI can also improve data security. As the data are indexed in a Single Identity Number (SIN), their use can be monitored. An unauthorised usage can also be detected as the system is integrated with the demographic database. Another improvement in terms of data security is in the transaction. The transaction that is executed with the approval of the account created by SIN will be able to prevent anonymity. The transaction will be saved along with passengers' identities who do transactions.

\subsection{Underlying Concept of Airline Ticket Reservation System Using Single Identity}

Airline ticket reservations using SI (e-KTP) can be completed using both automatic ticketing machines and online systems as can be seen in Figure 2. Passengers first access the system and select departure and arrival destinations. Then, they are required to tap their e-KTP on a machine or a smartphone, so that the system can retrieve all related data contained in the e-KTP. After that, passengers may proceed to select the names of the passengers registered in the Family Card. Passengers then need to confirm the details of the reservations and make a payment. In addition, the booking reference can be either printed out or stored in the NFC tag and/or in the NFC device. Retrieved data will comprise identity numbers, thus, they represent passenger data.

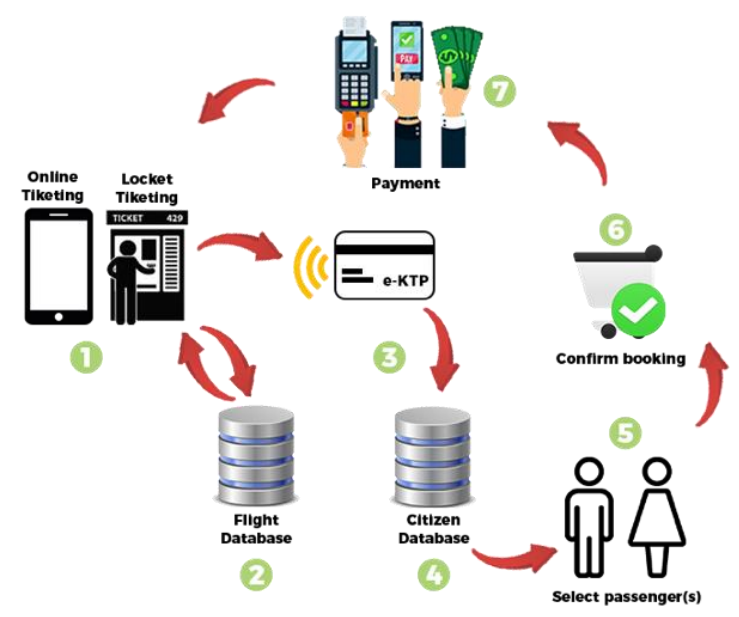

Figure 2. Flowchart of the application of SI in the airline ticketing reservation system

\subsection{Airline Ticket Reservation Using NFC-Based Single Identity}

Another advantage of using SI is an increase in the transaction speed. Time required for ticket reservations via ticket counters and online is different, resulting in different calculations. Regarding reservations in ticket counters, there is a time variable needed by passengers to get to the counters, while online reservations do not require such variables. Moreover, data input in ticket counters is done by the relevant staff, whereas passengers are required to enter their data by themselves. While making a payment in ticket counters can be completed by cash, debit, or credit cards; online reservations require passengers to purchase using online payments. Also, passengers receive a printed booking reference directly from the staff in ticket counters whereas in online reservations, booking reference is sent to passengers' email.

To do transactions, there are variables influencing the transaction speed: the time required to get to counters $\left(\mathrm{T}_{\mathrm{go}}\right)$, the time required to select arrival and departure destinations, and arrival and departure dates (arrival and departure for a round trip) and other additional information $\left(\mathrm{T}_{\text {fillform }}\right)$, the time required to retrieve flight schedules ( $\left.\mathrm{T}_{\text {schedule}}\right)$, the time required to input passenger data according to the number of passengers $\left(\mathrm{N}_{2} \mathrm{~T}_{\text {input }}\right)$, the time required to tap the e-KTP $\left(\mathrm{Tt}_{\mathrm{ap}}\right)$, the time required to send data through the internet $\left(T_{\text {submit }}\right)$, the time required for payments $\left(\mathrm{T}_{\text {pay }}\right)$, the time required to confirm reservations $\left(\mathrm{T}_{\text {confirm }}\right)$, the time required to receive the booking reference $\left(\mathrm{T}_{\text {receive }}\right)$. The use of self-service ticketing machines may substitute the role of humans (in this case, airport staff) in ticket reservation systems, so that the calculation for reservation systems 
in both ticket counters and online can be standardised. In general, to calculate the time needed to purchase tickets via machines and online, the formula is:

$$
\mathrm{T}_{\text {reservation }}=\mathrm{T}_{\text {go/access }}+\mathrm{T}_{\text {fillform }}+\mathrm{T}_{\text {schedule }}+\left[\left(\mathrm{T}_{\text {Id }}+\mathrm{T}_{\text {name }}\right) \mathrm{x} \mathrm{N}\right]+\mathrm{T}_{\text {confirm }}+\mathrm{T}_{\text {submit }}+\mathrm{T}_{\text {pay }}+\mathrm{T}_{\text {print/send }}
$$

Thus,

$$
\mathrm{T}_{\text {reservation }}=\mathrm{T}_{\text {go/access }}+\mathrm{T}_{\text {fillform }}+\mathrm{T}_{\text {schedule }}+\left(2 \mathrm{~T}_{\text {input }} \mathrm{x} \mathrm{N}\right)+\mathrm{T}_{\text {confirm }}+\mathrm{T}_{\text {submit }}+\mathrm{T}_{\text {pay }}+\mathrm{T}_{\text {print/send }}
$$

$\mathrm{T}_{\mathrm{go}}$ depends on both passengers' initial positions and the locations of ticket counters, thus, its calculation is overlooked. The process of filtering flight schedules requires several parameters, that is, departure dates, departure places, and return dates (for a round trip). Data input can be completed by searching and selecting relevant data, hence, the time required to complete passengers' preferences regarding flight schedules can be obtained by multiplying the total amount of data and the time needed to select $\mathrm{T}_{\text {select }}$.

$$
\mathrm{T}_{\text {fillform }}=\mathrm{Mx} \mathrm{T}_{\text {select }}
$$

The time required to send data and receive them relies on the internet access and the server, so in this calculation, $\mathrm{T}_{\text {access }}, \mathrm{T}_{\text {schedule }}$ and $\mathrm{T}_{\text {submit }}$ are omitted. The formula used to calculate the time needed for purchasing tickets via self-service ticketing machines and online is:

$$
\mathrm{T}_{\text {reservation }}=\mathrm{T}_{\text {fillform }}+\left(2 \mathrm{~T}_{\text {input }} \mathrm{x}\right)+\mathrm{T}_{\text {confirm }}+\mathrm{T}_{\text {pay }}+\mathrm{T}_{\text {print/send }}
$$

Using the e-KTP by tapping it on a machine or a smartphone requires time, that is, $\mathrm{T}_{\text {tap. }}$ NFC has the ability to read data faster, resulting in less time needed in the process of reading data. However, one of the possible constraints is that the e-KTP should be tapped right on the chip that will help read the NFC tag, and the position of the e-KTP should be within that distance. Hence, a longer time might be needed due to the positioning of e-KTP. The time required to read the NFC tag (e-KTP) is usually less then one (1) second, whereas the time required to position the NFC tag is around 2-3 seconds. Therefore, the total amount of time needed to read the NFC tag is:

$$
\mathrm{T}_{\text {tap }}=\mathrm{T}_{\text {read }}+\mathrm{T}_{\text {positioning }}
$$

Using the (3), (4), (5) equations, the calculation of ticket reservations utilising SI in the e-KTP is:

$$
\mathrm{T}_{\text {reservation }}=\left(\mathrm{M} \times \mathrm{T}_{\text {select }}\right)+\left(\mathrm{T}_{\text {read }}+\mathrm{T}_{\text {positioning }}\right)+\left(2 \mathrm{~T}_{\text {input }} \times \mathrm{N}\right)+\mathrm{T}_{\text {confirm }}+\mathrm{T}_{\text {pay }}+\mathrm{T}_{\text {print/send }}
$$

As the process of data input such as, passengers' names and identities can be done simultaneously, the time $2 \mathrm{~T}_{\text {input }}\left(\mathrm{T}_{\mathrm{Id}}+\mathrm{T}_{\text {name }}\right)$ can be calculated once, thus, the time required only depends on the number of passengers. The formula is:

$$
\mathrm{T}_{\text {reservation }}=\left(\mathrm{Mx} \mathrm{T}_{\text {select }}\right)+\left(\mathrm{T}_{\text {read }}+\mathrm{T}_{\text {positioning }}\right)+\mathrm{N}+\mathrm{T}_{\text {confirm }}+\mathrm{T}_{\text {pay }}+\mathrm{T}_{\text {print/send }}
$$

The assumption regarding the comparison of the time needed by four (4) ticket reservation methods, that is, ticket counters or automatic ticketing machines and online; and the comparison of these method with and without SI can be seen in Table 1.

Table 1. Assumption regarding the comparison of the time needed by ticket reservation methods (in seconds)

\begin{tabular}{lcccc}
\hline & \multicolumn{2}{c}{ Without SI (e-KTP) } & \multicolumn{2}{c}{ With SI (e-KTP) } \\
\cline { 2 - 5 } & Machine & Online & Machine & Online \\
\hline $\mathrm{T}_{\text {select }}$ & 5 & 5 & 5 & 5 \\
$\mathrm{~T}_{\text {read }}$ & 0 & 0 & 1 & 1 \\
$\mathrm{~T}_{\text {positioning }}$ & 0 & 0 & 4 & 4 \\
$\mathrm{~T}_{\text {confirm }}$ & 20 & 20 & 20 & 20 \\
$\mathrm{~T}_{\text {pay }}$ & 90 & 90 & 90 & 90 \\
$\mathrm{~T}_{\text {printsend }}$ & 10 & 20 & 5 & 20 \\
$\mathrm{~T}_{\text {input }}$ & 20 & 20 & 0 & 0 \\
\hline
\end{tabular}


The results of using the (6) and (7) equations to calculate the comparison of the time required by the four (4) ticket reservation methods can be seen in Figure 3.

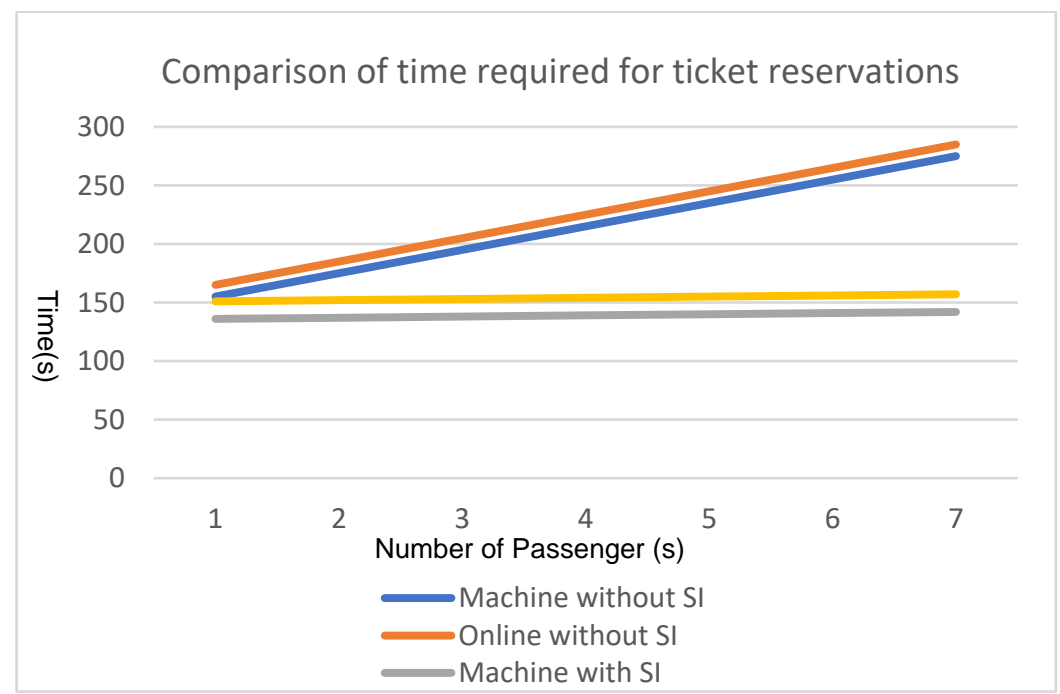

Figure 3. Comparison of time required for ticket reservations

The application of SI in ticket reservation systems using machines or online may lead to the time efficiency up to almost 50\%. The figure showing the efficiency can be seen in Figure 4.

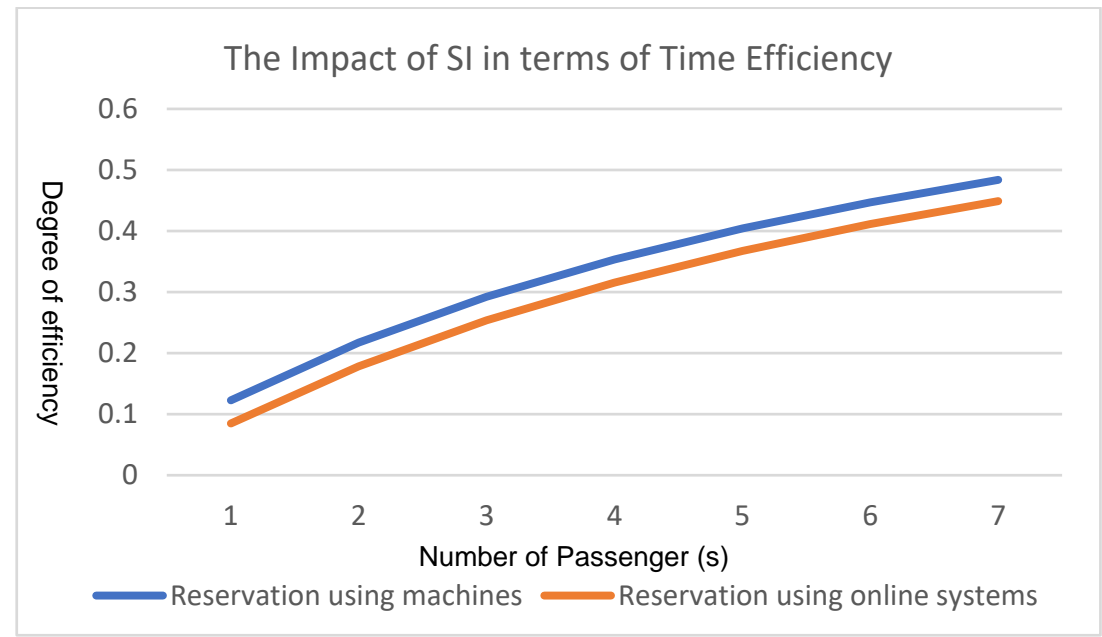

Figure 4. Time efficiency level towards the number of passengers

\section{CONCLUSION}

This study aims at implementing SI in airline ticket reservations. This implementation is to reduce the redundancy caused by the same data or different writings for the same data. By using SI numbers, all resident data attached in the same Family Card can be retrieved. Family data retrieved from the database can be utilised to purchase airplane tickets. An individual might not memorise his/her own SI numbers and his/her family members' SI numbers. Therefore, one does not need to concern if he/she forgets the other family members' SI numbers. Additionally, the application of SI can also speed up the transaction process and improve the data usage security. One of the drawbacks of this implementation is that tickets cannot be purchased by an individual who is not a member of of the family, such as travel agents. Further research may discuss validation methods such as, PIN or fingerprint might be required to ensure that the e-KTP is only used by the owner. 


\section{ACKNOWLEDGEMENTS}

We would like to thank Ministry of Research, Technology and Higher Education of the Republic of Indonesia and Bandung Institute of Technology for the research funding through Penelitian Terapan Unggulan Perguruan Tinggi 2018.

\section{REFERENCES}

[1] R. W. Dedy, "Middleware Architecture For National Electronic ID Card: The Case of E-KTP In Indonesia", Journal of Theoretical and Applied Information Technology, vol. 61, pp. 670-674, March 2014.

[2] S. Triyuni, "The Dynamic of e-KTP Evaluation Program in DKI Jakarta", International Journal of Administrative Science \& Organization, vol. 20, pp. 90-97, May 2013.

[3] Syaruddin, et al., "Policy Implementation of Public Service Department Civilian Population and Notes District Merauke", Journal of Research in Humanities and Social Science, vol. 3, pp. 8-12, 2015.

[4] M. R. Waghe, P. A. Pawar, S.N. Bhadane, "Use of NFC Technology In Electronic Ticket System For Public Transport", International Journal Of Engineering And Computer Science, vol. 3, pp. 5273-5274, April 2014.

[5] M. A. Ali, "An Innovative Approach for E-Goverment Transformation", International Journal of Managing Value and Supply Chains, vol 2, pp. 22-43, 2011.

[6] M. A. Ali, F. Muhammad, Q. Jameel, "A goverment framework to address identity, trust and security in e-goverment: The case of UAE identity management infrastructure”, European Scientific Journal, vol. 10, pp. 85-98, 2014.

[7] P. K. Tee, G. Behrooz, C.Y.F. Benjamin, "Electronic Ticketing in Airline Industries Among Malaysians: The Determinants", International Journal of Bussiness and Social Science, vol. 5, pp. 168-174, 2014.

[8] M. Deepak, "Drivers and Inhibitors in Online Ticketing in Civil Aviation Sector", Civil and Environmental Research, vol. 3, pp. 156-165, 2008.

[9] S. Ainin, N. Josephine, M. Suhana, "E-Ticketing As A New Way of Buying Tickets: Malaysian Perceptions", Journal Social Science, vol. 17, pp. 149-157, 2012.

[10] K. Q. Mazen, H. A. Haitham, A. A. Mohammad, "The Impact of E-Ticketing Technique on Customer Satisfaction: An Empirical Analysis", Journal of Information System and Technology Management, vol. 11, 2014.

[11] S. Wayan, "Application of Near Field Communication Technology for Mobile Airline Ticketing", Journal of Computer Science, vol. 8, pp. 1235-1243, 2012.

[12] C. Kevin, M. Amanda, M. G. Conor, "Near Field Communication", International Journal of Electrical and Computer Engineering (IJECE), vol. 2, 2012.

[13] B. C. Ugo, et al., "Mobile Ticketing With NFC Management for Transport Companies: Problems and Solutions", Paper presented at: CATTID (Centre for Application of Teleservices and Technologies for Innovation in Digital world) Sapienza University of Rome, 2013.

[14] Z. Dino, "Implementation Model for Near Field Communication in Croation Ferry Ticketing System", Procedia Engineering, vol 100, pp. 1396-1404, 2015.

[15] G. Subamarekha, et al., "Android Application for Ticket Booking and Ticket Checking in Suburban Railways", Indian Journal of Science and Technology, vol. 8, pp. 171-178, January 2015.

[16] L. Monalisa, et al., "Secure Bus-Ticketing System using NFC", International Journal of Engineering Research and Applications, vol. 5, pp. 43-45, April 2015.

[17] D. Sunita, et al., "NFC Based Technology for Railway Ticketing System", International Journal of Computing and Technology, vol. 1, pp. 467-469, October 2014.

[18] B. R. Mikhael, et al., "Baggage Claim in Airport Using Near Field Communication", Indonesian Journal of Electrical Engineering and Computer Science, vol. 7, pp. 442-448, 2017.

[19] B. Noor Cholis, et al., "Developing Electronic Medical Record Based on NFC", Paper presented at: International Conference on Computer Science and Artificial Intelligence, 2017.

[20] B. R. Mikhael, et al., "Securing Electronic Medical Record in Near Field Communication Using Advanced Encryption Standard (AES)", IOS Press on Technology and Health Care, vol. 26, pp. 357-362, 2018.

[21] H. M. Emir, Kuspriyanto, B. Noor Cholis, "Mobile Payment Protocol Tag-to-tag Near Field Communication (NFC)", iJIM, vol. 6, pp. 34-38, 2012.

[22] H. M. Emir, et al., "Near Field Communication (NFC) Protocol Using tag for Secure Mobile Payment", Paper presented at: Intelligent Technology and Its Application (SITIA) Seminar, 2012.

[23] H. M. Emir, et al., "Efficient tag-to-tag Near Field Communication (NFC) Protocol for Secure Mobile Payment", Paper presented at: ICICI-BME, 2011.

[24] S. Amarjeet, K. P. Ramakanth, G. C. Nagaraj, "Empowering E-governance with E-voting", Indonesian Journal of Electrical Engineering and Computer Science, vol. 12, pp. 1081-1086, 2018.

[25] A.B. Hajira Be, R. Balasubramanian, "Developing an Enhanced High-Speed Key Transmission (EHSKT) Technique To Avoid Fraud Activity in E-Commerce", Indonesian Journal of Electrical Engineering and Computer Science, vol. 12, pp. 1187-1194, 2018.

[26] Chadrakala, S.C.Lingareddy, "Secure And Efficient Bi-Directional Proxy Re-Encryption Technique", Indonesian Journal of Electrical Engineering and Computer Science, vol. 12, pp. 1143-1150, 2018. 


\section{BIOGRAPHIES OF AUTHORS}

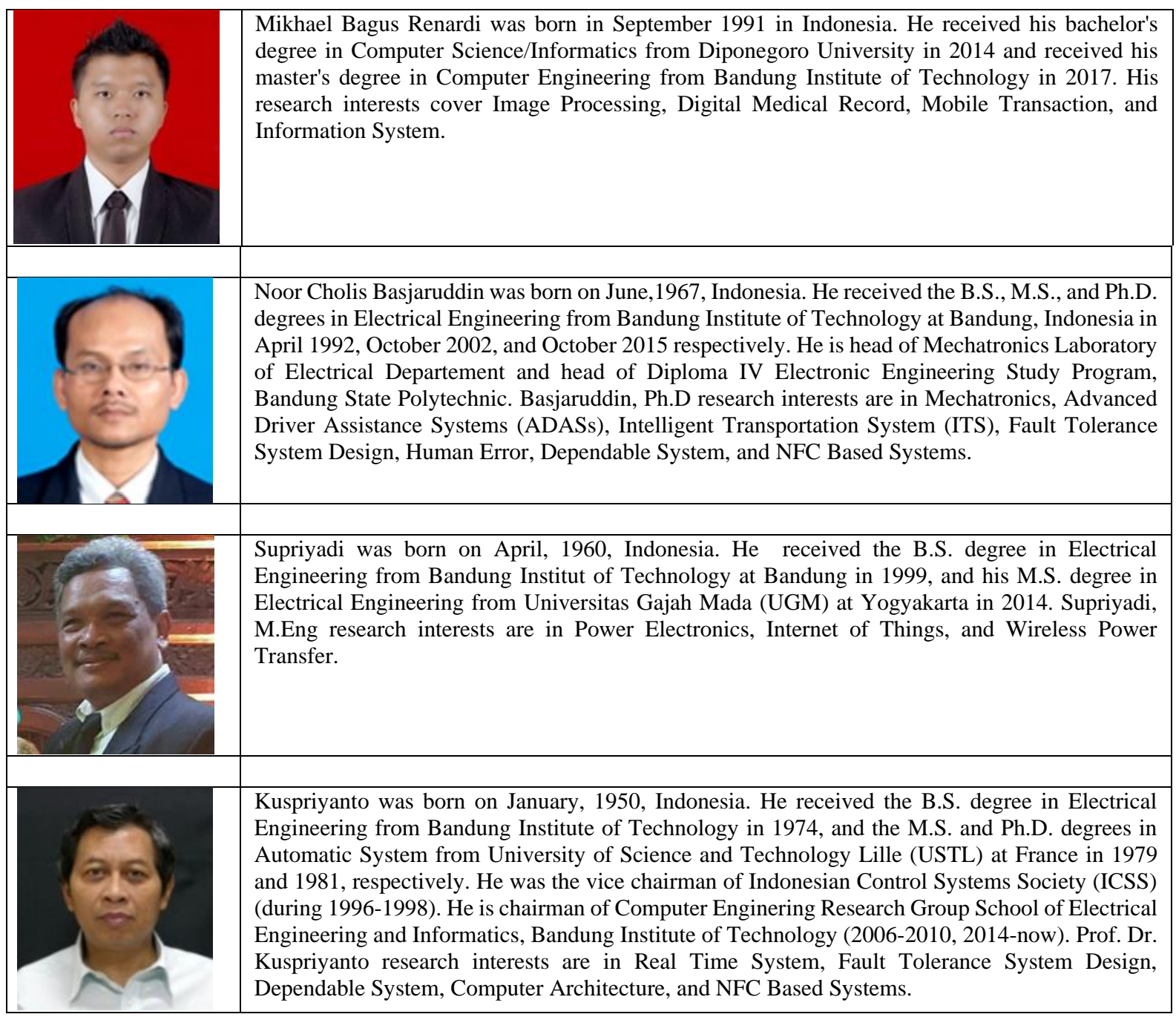

